\title{
Editorial
}

\section{Comparing Particle Productions at RHIC and LHC Energies}

\author{
Fu-Hu Liu, ${ }^{1}$ Sakina Fakhraddin, ${ }^{2}$ Edward Sarkisyan-Grinbaum, ${ }^{3,4}$ and Bhartendu K. Singh ${ }^{5}$ \\ ${ }^{1}$ Institute of Theoretical Physics, Shanxi University, Taiyuan, Shanxi 030006, China \\ ${ }^{2}$ Physics Department, Faculty of Science, Sana'a University, P.O. Box 1247, Sana'a, Yemen \\ ${ }^{3}$ Department of Physics, CERN, 1211 Geneva 23, Switzerland \\ ${ }^{4}$ Department of Physics, The University of Texas at Arlington, Arlington, TX 76019, USA \\ ${ }^{5}$ Physics Department, Banaras Hindu University, Varanasi 221 005, India \\ Correspondence should be addressed to Fu-Hu Liu; fuhuliu@163.com
}

Received 1 March 2015; Accepted 1 March 2015

Copyright (C) $2015 \mathrm{Fu}-\mathrm{Hu}$ Liu et al. This is an open access article distributed under the Creative Commons Attribution License, which permits unrestricted use, distribution, and reproduction in any medium, provided the original work is properly cited. The publication of this article was funded by $\mathrm{SCOAP}^{3}$.

The Relativistic Heavy-Ion Collider (RHIC) at Brookhaven National Laboratory (BNL) has been opening a new era for high energy collisions, in which the center-of-mass energy per nucleon pair reached highly $200 \mathrm{GeV}$. At the RHIC, a lot of experimental data on proton-proton, deuteron-gold, coppercopper, and gold-gold collisions at different $\mathrm{GeV}$ energies have been reported. The Large Hadron Collider (LHC) at European Organization for Nuclear Research (CERN) has advanced the center-of-mass energy per nucleon pair to $\mathrm{TeV}$ region. Many experimental pieces of data on protonproton, proton-lead, and lead-lead collisions at different $\mathrm{TeV}$ energies have been published. Correspondingly, many modeling works have been done on the data analyses at RHIC and LHC energies.

There are some common laws on particle productions in collisions at RHIC and LHC energies. Besides, more special properties are observed on the particle productions in the collisions. We are interested in comparative studies on the particle productions in collisions at RHIC and LHC energies. This is an important issue for cosmic ray physics also because more particles are produced in cosmic ray-induced collisions at higher energy. Comparative studies on the particle productions in collisions at RHIC, LHC, and higher energies are useful for high energy, nuclear, and cosmic ray physicists. Naturally, the related results can be compared with collisions at the Super Proton Synchrotron (SPS) at CERN.

This special issue concerns many topics in comparing particle productions at RHIC and LHC energies, for example, rapidity distributions and correlations, transverse momentum distributions and correlations, collective flow effects and correlations, critical behavior amidst minijets, extracted freeze-out parameters, statistical and dynamical fluctuations, dynamical evolution of interacting system, statistical behaviors of particle productions, space structures of interacting system, nuclear penetrating and stopping effects, nuclear viscidity effects, and nuclear shadowing effects.

The paper "Recognizing Critical Behavior amidst Minijets at the Large Hadron Collider" reviews two approaches in the diagnostics of critical behavior in heavy-ion collisions and considered the effects of minijets. The first approach is a local one which is concerned with the hadronization such as the multiplicity fluctuations, transverse momentum spectra, and azimuthal anisotropy at a local point in (pseudorapidity $\eta$, azimuth $\phi$ ) space. The second approach is a nonlocal one which has to do with quark-hadron phase transition where collective behavior through near-neighbor interaction can generate patterns of varying sizes in the $(\eta, \phi)$ space. Scaling behaviors are found in both approaches, based on theoretical calculations in accordance with the Ginzburg-Landau theory of phase transition and Ising model simulations. Particularly, the author suggests that the existing data from LHC have sufficient multiplicities in small transverse momentum intervals to make the observation of distinctive features of clustering of soft particles feasible, as well as voids, which characterize the critical behavior at phase transition from quarks to hadrons, without any ambiguity posed by the clustering of jet particles. 
The paper "Freeze-Out Parameters in Heavy-Ion Collisions at AGS, SPS, RHIC, and LHC Energies" reviews the chemical and kinetic freeze-out conditions in high energy heavy-ion collisions. Chemical freeze-out parameters are obtained using produced particle yields in central collisions while the corresponding kinetic freeze-out parameters are obtained using transverse momentum distributions of produced particles. For chemical freeze-out, different freezeout scenarios are discussed such as single and double/flavor dependent freeze-out surfaces. Kinetic freeze-out parameters are obtained by doing hydrodynamic inspired blast-wave fit to the transverse momentum distributions. The authors find weak dependence of the extracted chemical freeze-out parameters on the factors such as choice of thermodynamic ensemble and choice of free parameters and fitting procedures. The choice of chemical freeze-out scheme seems to influence the fitted thermal parameters significantly, and strange to nonstrange particle ratios are most sensitive to the chosen chemical freeze-out scheme. The barometric observable, transverse energy per charged particle is related to the chemical freeze-out. The kinetic freeze-out temperature suggests a decrease from lower to higher energies.

The paper "A Review on $\phi$ Meson Production in HeavyIon Collision" reviews the measurements of $\phi$ meson production in heavy-ion experiments. The energy dependence of $\phi$ meson invariant yield and the production mechanism, strangeness enhancement, parton energy loss, and partonic collectivity in nucleus-nucleus collisions are discussed. Effect of later stage hadronic rescattering on elliptic flow of proton is also discussed relative to corresponding effect on elliptic flow of $\phi$ meson. It is shown that the coalescence of positive and negative kaons is not the dominant production mechanism for $\phi$ meson in high energy heavy-ion collisions. The study of $\Omega / \phi$ and comparison to quark recombination model calculations indicate the $\phi$ mesons are produced via coalescence of thermalized strange quarks in collisions at $19.6 \mathrm{GeV}$ and above. The observed $\phi$ meson enhancement (unaffected by Canonical suppression effects) in heavy-ion collisions suggests that strangeness enhancement is due to formation of a dense partonic medium. The nuclear modification factor and elliptic flow measurements for $\phi$ mesons indicate the formation of partonic media in heavy-ion collisions at $19.6 \mathrm{GeV}$ and above energies, while hadronic interactions dominate in collisions at $11.5 \mathrm{GeV}$ or lower energies.

In the paper "Pion Transverse Momentum Spectrum, Elliptic Flow, and Interferometry in the Granular Source Model for RHIC and LHC Heavy Ion Collisions," the authors systematically investigate the pion transverse momentum spectrum, elliptic flow, and HBT (Hanbury Brown and Twiss) interferometry in $\mathrm{Au}$-Au collisions at $200 \mathrm{GeV}$ and $\mathrm{Pb}-\mathrm{Pb}$ collision at $2.76 \mathrm{TeV}$ with different centralities by using a granular source model. It is shown that the model can well reproduce the experimental results of heavy-ion collisions at the RHIC and LHC. The experimental data of the momentum spectrum, elliptic flow, and HBT radii for the two collision energies and different centralities impose very strict constraints on the model parameters. They exhibit certain regularities for collision centrality and energy. The space-time structure and expansion velocities of the granular sources for the heavy-ion collisions at RHIC and LHC energies with different centralities are investigated.

In the paper "Production of Kaon and $\Lambda$ in NucleusNucleus Collisions at Ultrarelativistic Energy from a BlastWave Model," the authors employ the blast-wave model with a chemical equilibrium model to calculate the production of Kaon and $\Lambda$ in nucleus-nucleus collisions at relativistic energy. The baryon chemical potential and chemical freezeout temperature are obtained to be consistent with those extracted from experiments and used in other model calculations. The kinetic freeze-out properties such as kinetic freeze-out temperature and radial flow parameter are also discussed. These kinetic freeze-out parameters are in good agreement with experimental results and are found to be valid for strangeness hadrons (Kaon and $\Lambda$ ). In addition, the resonance decay effect on strangeness production is also studied in detail. The present work shows that the beam energy dependence of Kaon and $\Lambda$ production can be a good probe to study the properties of the dense matter created in ultrarelativistic heavy-ion collisions.

In the paper "A Systematic Study of Magnetic Field in Relativistic Heavy-Ion Collisions in the RHIC and LHC Energy Regions," the authors systematically study the features of magnetic field in relativistic heavy-ion collisions by using a modified magnetic field model. The features of magnetic field distributions in the central point are studied in the RHIC and LHC energy regions. Based on the detailed study at RHIC energies, the features of magnetic fields at higher LHC energies are predicted. The dependencies of the features of magnetic fields on the collision energy, centrality, and collision time are systematically investigated, respectively. It is shown that an enormous magnetic field can be created in off-central heavy-ion collisions. The magnitude of the field is quite large in the beginning of the collisions and decreases rapidly with time. Comparing with that in RHIC energy region, the magnitude of the magnetic fields with proper time drops more rapidly in LHC energy region.

In the paper "Properties of Full Jet in High-Energy Heavy-Ion Collisions from Parton Scatterings," the properties of fully reconstructed jets produced in $p+p$ and $\mathrm{Pb}+\mathrm{Pb}$ collisions at $2.76 \mathrm{TeV}$ are investigated by the authors within a multiphase transport (AMPT) model with both partonic scatterings and hadronic rescatterings. A large transverse momentum asymmetry of dijet or photon-jet arises from the strong interactions between jet and partonic matter. The jet fragmentation function in $\mathrm{Pb}+\mathrm{Pb}$ collisions is decomposed into two contributions from different jet hadronization mechanisms, that is, fragmentation versus coalescence. The medium modification of differential jet shape displays that the jet energy is redistributed towards a larger radius owing to jet-medium interactions. Jet triangular azimuthal anisotropy coefficient shows a smaller magnitude than the elliptic coefficient and decreases more quickly with increase of jet transverse momentum.

In the paper "Importance of Nonperturbative QCD Parameters for Bottom Mesons," the authors discuss the importance of nonperturbative quantum chromodynamics (QCD) parameters in context to the predicting power for bottom meson masses and isospin splitting. In the framework 
of heavy quark effective theory, the work presented in this paper focuses on the different allowed values of the two nonperturbative QCD parameters used in heavy quark effective theory formula. In addition, using the best fitted parameter, masses of the excited bottom meson states in $j^{p}=$ $(1 / 2)^{+}$doublet in strange as well as nonstrange sector are calculated. The calculated masses are found to be matching well with experiments and other phenomenological models. The mass and hyperfine splitting are also analyzed for both strange and nonstrange heavy mesons with respect to spin and flavor symmetries.

In the paper "The Profile of Inelastic Collisions from Elastic Scattering Data," by using the unitarity relation in combination with experimental data about the elastic scattering in the diffraction cone, the author studies how the shape and the darkness of the inelastic interaction region of colliding protons change with increase of their energies. In the framework of the indubitable unitarity condition using experimental data about the elastic scattering of protons in the diffraction cone without any reference to QCD or phenomenological approaches, some interesting conclusions are obtained. Particularly, the collisions become fully absorptive at small impact parameters at LHC energies that result in some special features of inelastic processes. Possible evolution of this shape with the dark core at the LHC to the fully transparent one at higher energies is discussed that implies the terminology of the black disk would be replaced by the black toroid. The approach to asymptotics is disputed. The ratio of the real to imaginary parts of the nonforward elastic scattering amplitude is briefly discussed.

The paper "Pion $p_{T}$ Spectra in $p+p$ Collisions as a Function of $\sqrt{s}$ and Event Multiplicity" studies the charged pion transverse momentum (or transverse mass) spectra in $p+p$ collisions by using the Tsallis distribution. These transverse spectra can be regarded as a function of collision energy $\sqrt{s}$ and event multiplicity. This study covers pion transverse spectra measured in $p+p$ collisions at SPS, RHIC, and LHC energies and gives an insight of the pion production process in the collisions. The Tsallis parameters are obtained and parameterized as a function of $\sqrt{s}$. It is suggested that more hard processes contribute to the transverse spectra at higher energy. This paper also studies the charged pion spectra for different event multiplicities (rapidity density) in $p+p$ collisions for LHC energies by using the Tsallis distribution. The variation of the Tsallis parameters as a function of event multiplicity has been obtained, and their behavior is found to be independent of collision energy.

The paper "Energy Dependence of Slope Parameter in Elastic Nucleon-Nucleon Scattering" investigates the diffraction slope parameter for elastic proton-proton and protonantiproton scattering based on all available experimental data at low and intermediate momentum transfer values. Energy dependence of the elastic diffraction slope is approximated by various analytic functions. The expanded "standard" logarithmic approximations with minimum number of free parameters allow description of the experimental slopes in all available energy range reasonably. The estimations of asymptotic shrinkage parameter are obtained for various $|t|$ domains based on all available experimental data. Various approximations differ from each other in both low energy and very high energy domains. Predictions for diffraction slope parameter are obtained for elastic proton-proton scattering and for proton-antiproton elastic reaction in different energy domains for various approximation functions.

The paper "Azimuthally Integrated HBT Parameters for Charged Pions in Nucleus-Nucleus Interactions versus Collision Energy" studies the energy dependence of spatiotemporal characteristics of particle emission region for charged pions produced in nucleus-nucleus collisions. No dramatic change is observed for the HBT parameters with increasing of the center-of-mass energy per nucleon-nucleon pair from a few $\mathrm{GeV}$ to a few $\mathrm{TeV}$. The emission duration is obtained to be almost independent of the center-of-mass energy within the measurement uncertainties. The analytic function is suggested for a smooth approximation of the energy dependence of the main HBT parameters. The fits demonstrate reasonable agreement with the experimental data. Predictions are made for future experiments at higher energies.

The paper "The Evolution-Dominated Hydrodynamic Model and the Pseudorapidity Distributions in High Energy Physics" discusses the pseudorapidity distributions of the charged particles produced in high energy heavy-ion collisions in the context of evolution-dominated hydrodynamic model. The contributions of hot and dense matter created in the collisions and leading particles are taken into account in the model. It is shown that the effect of initial flow of the hot and dense matter is negligible, compared with the effect of pressure gradient. The motion of this matter is mainly governed by the evolution of fluid. With the scheme of Khalatnikov potential, this model can be solved exactly, and the rapidity distribution of charged particles can be expressed in a simple analytical form. The leading particles are supposed to have a Gaussian rapidity distribution normalized to the number of participants. The theoretical results are in agreement with the experimental data measured at the RHIC and LHC.

The paper "The Unified Hydrodynamics and the Pseudorapidity Distributions in Heavy Ion Collisions at BNLRHIC and CERN-LHC Energies" integrates the features of the two famous hydrodynamic models (Hwa-Bjorken and Landau) together, by generalizing the relation between ordinary rapidity and space-time rapidity. In case of linear equation of state, this hydrodynamic model can be solved analytically. The exact solutions can then be used to formulate the rapidity distributions of the charged particles frozen out from the fluid at the space-like hypersurface with a fixed proper time. It is shown that the unified hydrodynamics is successful in describing the rapidity distributions of charged pions, kaons, and antiprotons, where the contributions of leading particles have to be considered in proton spectra. The unified hydrodynamics and the evolution-dominated hydrodynamic model are mainly different in two ways: initial conditions and freeze-out conditions.

The paper "Transverse Momentum Spectra of $K_{S}^{0}$ and $K^{* 0}$ at Midrapidity in $d+\mathrm{Au}, \mathrm{Cu}+\mathrm{Cu}$, and $p+p$ Collisions at $\sqrt{s_{N N}}=200 \mathrm{GeV}^{\prime}$ compares the Tsallis statistics and the two-Boltzmann distribution in the analysis of the transverse momentum spectra of the mentioned particles produced 
in the mentioned collisions. It is shown that both the two methods can describe the distribution of the final-state particles. They have advantage and proper scope, respectively. The two forms of Tsallis distribution can consistently agree with the experimental points in the low and high transverse momentum region. The two-Boltzmann distribution can directly reflect the contributions of the soft interactions and the hard interactions in the observed spectra. The effective temperature extracted from the Tsallis distribution is lower than that extracted from the two-Boltzmann distribution. To describe the "real" temperature of emission source, both the distributions need to be revised.

In the paper "Nuclear Stopping in Central Au+Au Collisions at RHIC Energies," the authors present the pseudorapidity distribution of charged particles for central $\mathrm{Au}+\mathrm{Au}$ reactions at RHIC energies by using the UrQMD transport model (version 3:3) with the cascade mode and the modified mode (considering the potentials of both formed and "preformed" hadrons). Based on the model, the effect of mean-field potentials on nuclear stopping is investigated. It is found that nuclear stopping is increasingly influenced by the mean-field potentials in the projectile and target regions with increase of the reaction energy. Since most of the incident energy is concentrated in the central region, the calculations of the model with the cascade mode considered that the modifying factor can describe the experimental data in the projectile and target region. The modeling results are in agreement with the experimental data of central $\mathrm{Au}+\mathrm{Au}$ collisions at $62.4,130$, and $200 \mathrm{GeV}$.

In the paper "Chemical Potentials of Quarks Extracted from Particle Transverse Momentum Distributions in Heavy Ion Collisions at RHIC Energies," the authors investigate the transverse momentum distributions of charged particles produced in nucleus-nucleus and deuteron-nucleus collisions at RHIC energies by using a two-component revised Boltzmann distribution which is naturally considered in the framework of a multisource thermal model. The calculated results are in agreement with the PHENIX experimental data. It is found that the effective temperature increases obviously with increase of the particle mass and incident energy, but it does not show an obvious change with the collision centrality. The values of chemical potentials for up, down, and strange quarks are then obtained from the antiparticle to particle yield ratios in a wide transverse momentum range. The relationship between the chemical potentials of quarks and the transverse momentum with different centralities are investigated, too.

In the paper "Transverse Momentum and Pseudorapidity Distributions of Charged Particles and Spatial Shapes of Interacting Events in $\mathrm{Pb}-\mathrm{Pb}$ Collisions at $2.76 \mathrm{TeV}$," the authors analyze the transverse momentum and pseudorapidity distributions of charged particles produced in $\mathrm{Pb}-\mathrm{Pb}$ collisions with different centrality intervals at $2.76 \mathrm{TeV}$ by using the multisource thermal model in which the whole interacting system and then the sources are described by the Tsallis statistics. The modelling results are in agreement with experimental data of the ALICE Collaboration. The rapidity distributions of charged particles are obtained according to the extracted parameter values. The shapes of interacting events (the dispersion plots of charged particles) at the stage of kinetic freeze-out are given in the momentum, rapidity, velocity, and coordinate spaces. Meanwhile, the event shapes at the stage of kinetic freeze-out in different spaces consisting of different transverse quantities and longitudinal quantities are presented.

This issue brings together a collection of review and research papers on comparing particle productions at RHIC and LHC energies. We hope this will be a useful issue for researchers working in related areas. Meanwhile, we regret that more manuscripts submitted for publication in this issue have not been accepted according to reviewer's reports.

\section{Acknowledgment}

We sincerely thank all the authors who contributed to this issue.

$\mathrm{Fu}-\mathrm{Hu} \mathrm{Liu}$

Sakina Fakhraddin

Edward Sarkisyan-Grinbaum

Bhartendu K. Singh 

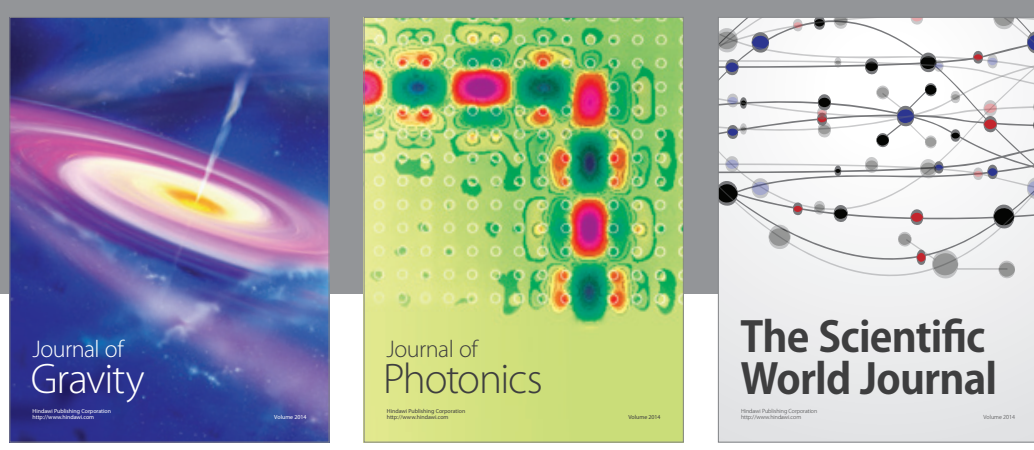

The Scientific World Journal
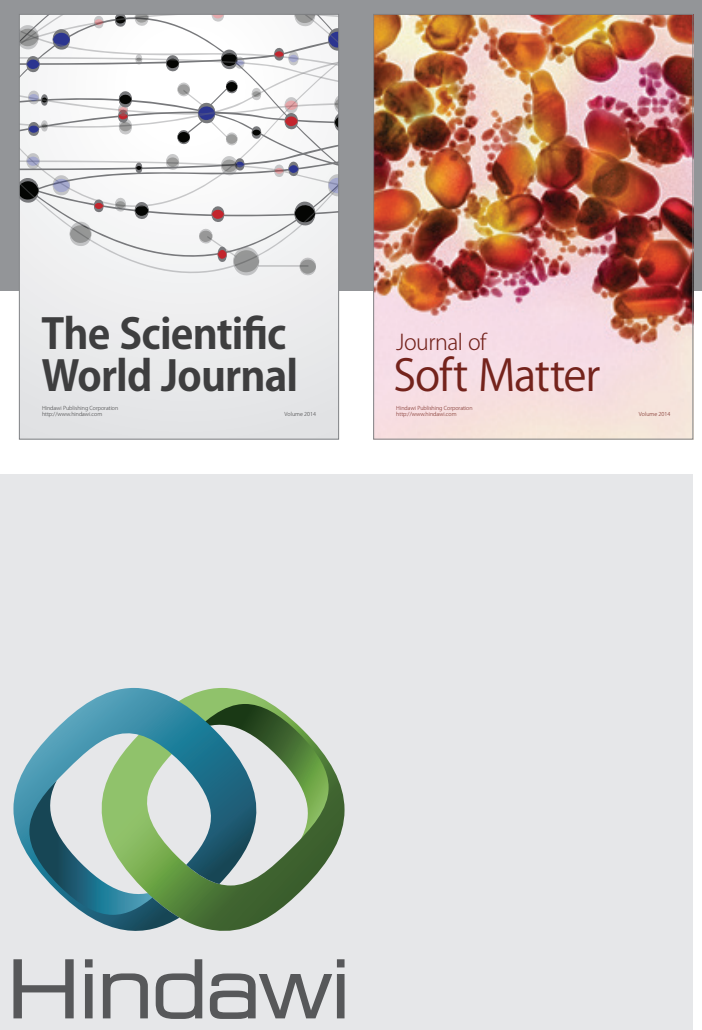

Submit your manuscripts at

http://www.hindawi.com

nternational Journal of

Statistical Mechanics
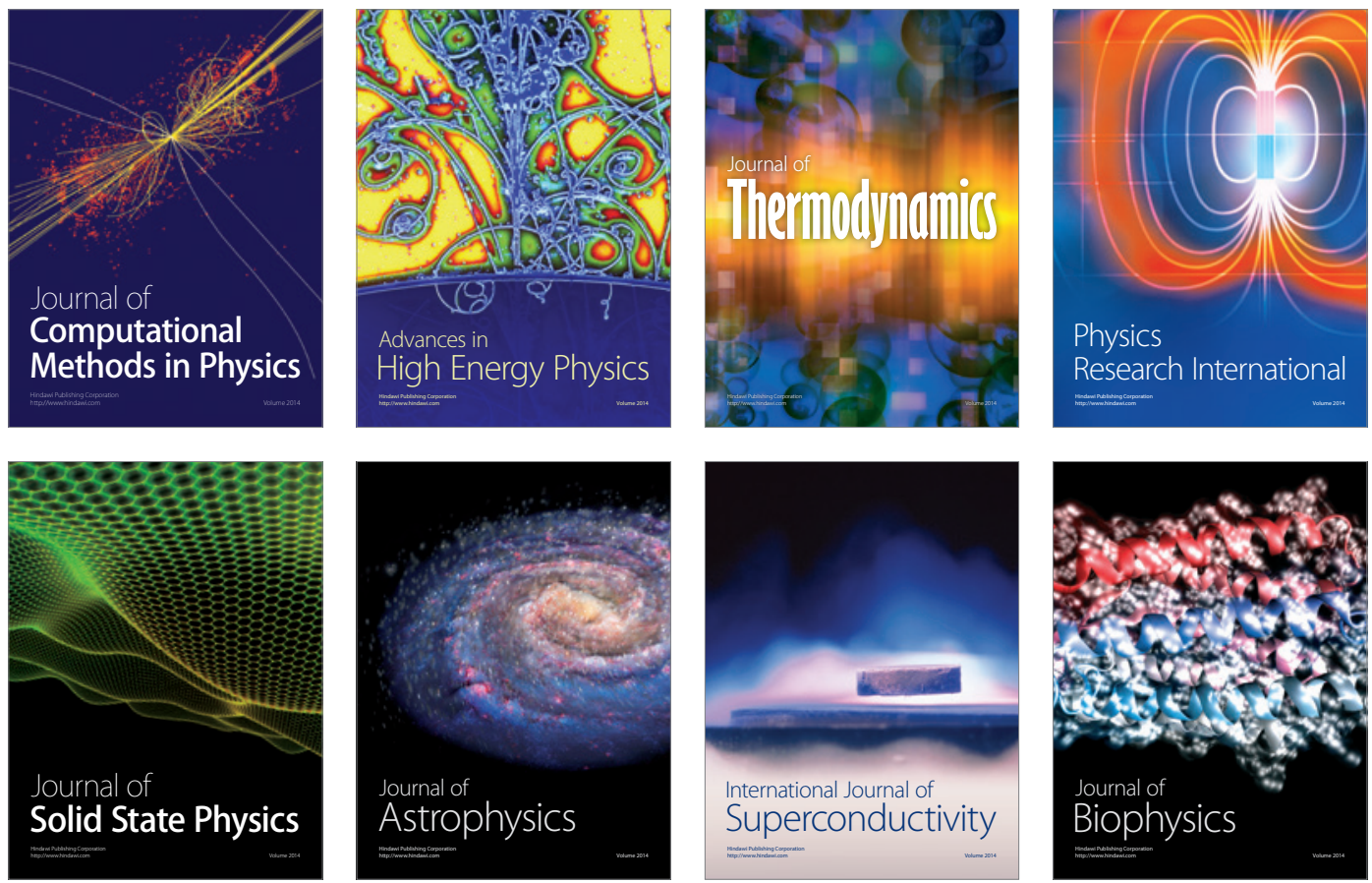
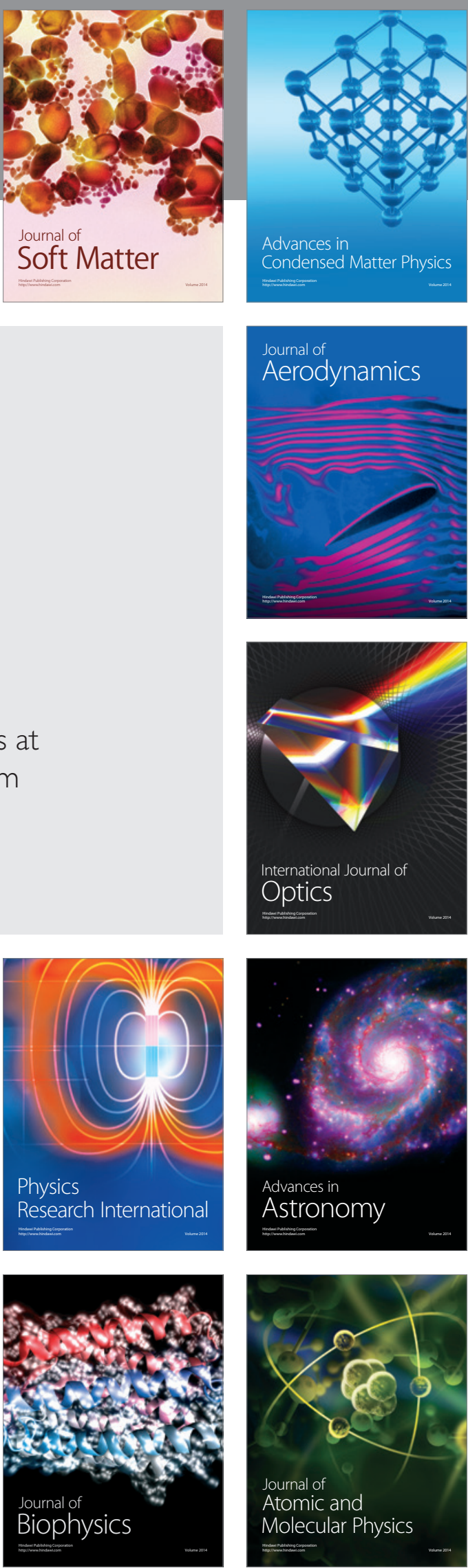